\title{
Reasoning in Expressive Description Logics under Infinitely Valued Gödel Semantics`
}

\author{
Stefan Borgwardt ${ }^{1}$ and Rafael Peñaloza ${ }^{2}$ \\ 1 Theoretical Computer Science, TU Dresden, Germany \\ Stefan.Borgwardt@tu-dresden.de \\ 2 KRDB Research Centre, Free University of Bozen-Bolzano, Italy \\ rafael.penaloza@unibz.it
}

\begin{abstract}
Fuzzy Description Logics (FDLs) combine classical Description Logics with the semantics of Fuzzy Logics in order to represent and reason with vague knowledge. Most FDLs using truth values from the interval $[0,1]$ have been shown to be undecidable in the presence of a negation constructor and general concept inclusions. One exception are those FDLs whose semantics is based on the infinitely valued Gödel tnorm (G). We extend previous decidability results for the FDL G- $\mathcal{A} \mathcal{L C}$ to deal with complex role inclusions, nominals, inverse roles, and qualified number restrictions. Our novel approach is based on a combination of the known crispification technique for finitely valued FDLs and an automata-based procedure for reasoning in $\mathrm{G}-\mathcal{A L C}$.
\end{abstract}

\section{Introduction}

Description Logics (DLs) are a well-studied family of knowledge representation formalisms [1]. They constitute the logical backbone of the standard Semantic Web ontology language OWL $2,{ }^{3}$ and its profiles, and have been successfully applied to represent the knowledge of many and diverse application domains, particularly in the bio-medical sciences. DLs describe the domain knowledge using concepts (such as Patient) that represent sets of individuals, and roles (hasChild) that represent connections between individuals. Ontologies are collections of axioms formulated over these concepts and roles, which restrict the possible interpretations. The typical axioms considered in DLs are assertions, like alice:Patient, providing knowledge about specific individuals; general concept inclusions (GCIs), such as Patient $\sqsubseteq$ Human, which express subset relations between concepts; and role inclusions hasChild o hasChild $\sqsubseteq$ hasGrandchild between (chains of) roles. Different DLs are characterized by the constructors allowed to formulate complex concepts, roles, and axioms.

$\mathcal{A L C}[30]$ is a prototypical DL of intermediate expressivity that uses as concept constructors: conjunction (Patient $\sqcap$ Female), negation ( $\neg$ Smoker), existential

\footnotetext{
* This work was partially supported by DFG grant BA 1122/17-1 'FuzzyDL', CRC 912 'HAEC', and the Cluster of Excellence 'cfAED', and developed while R. Peñaloza was affiliated with TU Dresden and the Center for Advancing Electronics Dresden.

${ }^{3}$ http://www.w3.org/TR/ow12-overview/
} 
restriction ( $\exists$ hasChild.HeavySmoker), and value restriction ( $\forall$ hasChild.Male), and allows assertions and GCIs. The DL underlying the OWL 2 DL standard is called $\mathcal{S R O I Q}$ and additionally provides, among others, role inclusions, number restrictions ( $\geqslant 3$ hasChild.Adult), nominals ( $\{$ alice $\}$ ), and inverse roles (hasChild ${ }^{-}$). The complexity of common reasoning problems, such as consistency of ontologies or subsumption between concepts, has been extensively studied for these DLs, and ranges from ExpTime to (CO-)2-NExpTime [26, 29,33].

Fuzzy Description Logics (FDLs) have been introduced as extensions of classical DLs to represent and reason with vague knowledge. The main idea is to use truth values from the interval $[0,1]$ instead of only true and false. In this way, one can give a more fine-grained semantics to inherently vague concepts like LowFrequency or HighConcentration, which can be found in biomedical ontologies like SNOMED CT ${ }^{4}$ and Galen. ${ }^{5}$ Different FDLs are characterized not only by the constructors they allow, but also by the way these constructors are interpreted. To interpret conjunction in complex concepts like

\section{$\exists$ hasHeartRate.LowFrequency $\sqcap$ \\ ᄏhasBloodAlcohol.HighConcentration,}

a popular approach is to use so-called t-norms [27]. The semantics of the other logical constructors can then be derived from these t-norms in a principled way, as suggested in [20]. Following the principles of mathematical fuzzy logic, existential and value restrictions are interpreted as suprema and infima of truth values, respectively. However, to avoid problems with infinitely many truth values, reasoning in fuzzy DLs is often restricted to so-called witnessed models [21], in which these suprema (infima) are required to be maxima (minima); i.e. the truth value of the restriction is witnessed by at least one domain element.

Unfortunately, most FDLs become undecidable when the logic uses GCIs and negation under witnessed model semantics $[2,13,18]$. One of the few exceptions are FDLs using the Gödel t-norm, which is defined as $\min \{x, y\}$, to interpret conjunctions [12]. In the absence of an involutive negation constructor and negated assertions, such FDLs are even trivially equivalent to classical DLs [13]. However, in the presence of the involutive negation, reasoning becomes more complicated. Despite not being as well-behaved as finitely valued FDLs, which use a finite total order of truth values instead of the infinite interval $[0,1]$, it was shown using an automata-based approach that reasoning in Gödel extensions of $\mathcal{A L C}$ exhibits the same complexity as in the classical case, i.e. it is EXPTimE-complete [12]. A major drawback of this approach is that it always has an exponential runtime, even when the input ontology has a simple form.

In the present paper, we present a combination of the automata-based construction for $\mathcal{A L C}$ from [12] and automata-based algorithms and reduction techniques developed for more expressive finitely valued FDLs [5, 6, 10,11, 14, 15, 31]. We exploit the forest model property of classical DLs $[17,25]$ to encode order

\footnotetext{
${ }^{4}$ http://www.ihtsdo.org/snomed-ct/

${ }^{5}$ http://www.opengalen.org/
} 
relationships between concepts in a fuzzy interpretation in a manner similar to the Hintikka trees from [12]. However, instead of using automata to determine the existence of such trees, we reduce the fuzzy ontology directly into a classical $\mathcal{A L C O Q}$ ontology, which enables us to use optimized reasoners for classical DLs. In addition to the cut-concepts of the form $C \geqslant p$ for a fuzzy concept $C$ and a value $p$, which are used in the reductions for finitely valued DLs [6,10,31], we employ order concepts $C \leqslant D$ expressing relationships between fuzzy concepts. The details of these concepts are explained in Section 4. In contrast to the reductions for finitely valued Gödel FDLs [6,7], our reduction does not produce an exponential blowup in the nesting depth of concepts in the input ontology.

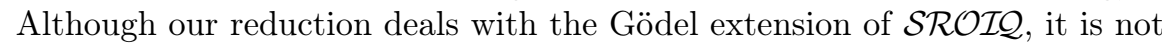
correct if all three constructors nominals $(\mathcal{O})$, inverse roles $(\mathcal{I})$, and number restrictions $(\mathcal{Q})$ are present in the ontology, since then one cannot restrict rea-

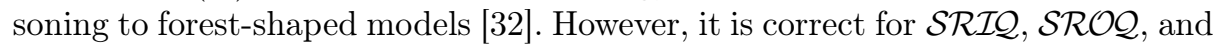
$\mathcal{S R O I}$, and we obtain several complexity results that match the currently best known upper bounds for reasoning in (sublogics of) these DLs. In particular, we show that reasoning in Gödel extensions of $\mathcal{S R I \mathcal { Q }}$ is 2-ExPTimE-complete, and for $\mathcal{S H O I}$ and $\mathcal{S H I Q}$ it is ExpTime-complete. Full proofs of all presented results can be found in [16].

\section{Preliminaries}

We consider vague statements taking truth degrees from the subset $[0,1]$ of the reals, where the Gödel t-norm $\min \{x, y\}$ is used to interpret logical conjunction. The semantics of implications is given by the residuum of this t-norm; i.e.,

$$
x \Rightarrow y:= \begin{cases}1 & \text { if } x \leqslant y \\ y & \text { otherwise }\end{cases}
$$

We use both the residual negation $x \mapsto(x \Rightarrow 0)$ and the involutive negation $x \mapsto(1-x)$ in the rest of this paper.

We recall some basic definitions from [12]. An order structure $S$ is a finite set containing at least the numbers $0,0.5$, and 1 , endowed with an involutive unary operation inv: $S \rightarrow S$ such that $\operatorname{inv}(x)=1-x$ for all $x \in S \cap[0,1]$. A total preorder over $S$ is a transitive and total binary relation $\preccurlyeq \subseteq S \times S$. For $x, y \in S$, we write $x \equiv y$ if $x \preccurlyeq y$ and $y \preccurlyeq x$. Notice that $\equiv$ is an equivalence relation on $S$. The total preorders considered in [12] have to satisfy additional properties, e.g. that 0 and 1 are always the least and greatest elements, respectively. These properties can be found in our reduction in the axioms of $\operatorname{red}(\mathcal{U})$ (see Section 4).

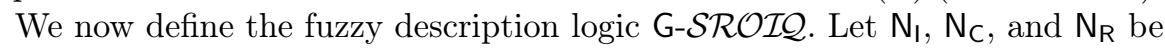
three mutually disjoint sets of individual names, concept names, and role names, respectively, where $\mathrm{N}_{\mathrm{R}}$ contains the universal role $r_{u}$. The set of (complex) roles is $\mathrm{N}_{\mathrm{R}}^{-}:=\mathrm{N}_{\mathrm{R}} \cup\left\{r^{-} \mid r \in \mathrm{N}_{\mathrm{R}}\right\}$; the elements of the form $r^{-}$are called inverse roles. Since there are several syntactic restrictions based on which roles appear in which role axioms, we start by defining role hierarchies. A role hierarchy $\mathcal{R}_{h}$ 
is a finite set of (complex) role inclusions of the form $\langle w \sqsubseteq r \geqslant p\rangle$, where $r \neq r_{u}$ is a role name, $w \in\left(\mathrm{N}_{\mathrm{R}}^{-}\right)^{+}$is a non-empty role chain not including the universal role, ${ }^{6}$ and $p \in(0,1]$. Such a role inclusion is called simple if $w \in \mathbf{N}_{\mathbf{R}}^{-}$. We extend the notation $\cdot^{-}$to inverse roles $r^{-}$and role chains $w=r_{1} \ldots r_{n}$ by setting $\left(r^{-}\right)^{-}:=r$ and $w^{-}:=r_{n}^{-} \ldots r_{1}^{-}$.

We recall the regularity condition from $[5,23]$. Let $\prec$ be a strict partial order on $\mathrm{N}_{\mathrm{R}}^{-}$such that $r \prec s$ iff $r^{-} \prec s$. A role inclusion $\langle w \sqsubseteq r \geqslant p\rangle$ is $\prec-r e g u l a r$ if

- $w$ is of the form $r r$ or $r^{-}$, or

$-w$ is of the form $r_{1} \ldots r_{n}, r r_{1} \ldots r_{n}$, or $r_{1} \ldots r_{n} r$, and for all $1 \leqslant i \leqslant n$ it holds that $r_{i} \prec r$.

A role hierarchy $\mathcal{R}_{h}$ is regular if there is a strict partial order $\prec$ as above such that each role inclusion in $\mathcal{R}_{h}$ is $\prec$-regular. A role name $r$ is simple (w.r.t. $\mathcal{R}_{h}$ ) if for each $\langle w \sqsubseteq r \geqslant p\rangle \in \mathcal{R}_{h}$ we have that $w$ is of the form $s$ or $s^{-}$for a simple role $s$. This notion is well-defined since the regularity condition prevents any cyclic dependencies between role names in $\mathcal{R}_{h}$. An inverse role $r^{-}$is simple if $r$ is simple. In the following, we always assume that we have a regular role hierarchy $\mathcal{R}_{h}$.

Concepts in G-SROIQ are built from concept names using the constructors listed in the upper part of Table 1 , where $C, D$ denote concepts, $p$ is a rational number from $[0,1], n \in \mathbb{N}, a \in \mathrm{N}_{\mathrm{l}}, r \in \mathrm{N}_{\mathrm{R}}^{-}$, and $s \in \mathrm{N}_{\mathrm{R}}^{-}$is a simple role. The restriction to simple roles in at-least restrictions is necessary to ensure decidability [24]. We also use the common DL constructors $T:=\overline{1}$ (top concept), $\perp:=\overline{0}$ (bottom concept), $C \sqcup D:=\neg(\neg C \sqcap \neg D)$ (disjunction), and $\leqslant n s . C:=\neg(\geqslant(n+1) s . C)$ (at-most restriction).

Note that we use the involutive negation to define at-most restrictions. In [7], they are defined using the residual negation: $\leqslant n s . C:=(\geqslant(n+1) s . C) \rightarrow \perp$. This has the effect that the value of $\leqslant n r . C$ is always either 0 or 1 (see the semantics below). However, this discrepancy in definitions is not an issue since our reduction can handle both cases. The use of rational truth constants $\bar{p}$ is not standard in FDLs, but it allows us to simulate fuzzy nominals [4] of the form $\left\{p_{1} / a_{1}, \ldots, p_{n} / a_{n}\right\}$ with $p_{i} \in[0,1]$ and $a_{i} \in \mathrm{N}_{\mathrm{l}}, 1 \leqslant i \leqslant n$, via $\left(\left\{a_{1}\right\} \sqcap \overline{p_{1}}\right) \sqcup \cdots \sqcup\left(\left\{a_{n}\right\} \sqcap \overline{p_{n}}\right)$.

The semantics of G-SROIQ is based on G-interpretations $\mathcal{I}=\left(\Delta^{\mathcal{I}},{ }^{\mathcal{I}}\right)$ over a non-empty domain $\Delta^{\mathcal{I}}$, which assign to each individual name $a \in N_{\text {I }}$ an element $a^{\mathcal{I}} \in \Delta^{\mathcal{I}}$, to each concept name $A \in \mathrm{N}_{\mathrm{C}}$ a fuzzy set $A^{\mathcal{I}}: \Delta^{\mathcal{I}} \rightarrow[0,1]$, and to each role name $r \in \mathrm{N}_{\mathrm{R}}$ a fuzzy binary relation $r^{\mathcal{I}}: \Delta^{\mathcal{I}} \times \Delta^{\mathcal{I}} \rightarrow[0,1]$. This interpretation is extended to complex concepts and roles as defined in the last column of Table 1 , for all $d, e \in \Delta^{\mathcal{I}}$.

We restrict all reasoning problems to witnessed G-interpretations [21], which intuitively require the suprema and infima in the semantics to be maxima and minima, respectively. Formally, a G-interpretation $\mathcal{I}$ is witnessed if, for every $d \in \Delta^{\mathcal{I}}, n \geqslant 0, r \in \mathrm{N}_{\mathrm{R}}^{-}$, simple $s \in \mathrm{N}_{\mathrm{R}}^{-}$, and concept $C$, there are

\footnotetext{
${ }_{6}$ For ease of presentation, we omit the composition symbol $\circ$ from role chains.
} 
Table 1. Syntax and semantics of G-SROIQ

\begin{tabular}{|c|c|c|}
\hline Name & Syntax & Semantics $\left(C^{\mathcal{I}}(d) / r^{\mathcal{I}}(d, e)\right)$ \\
\hline concept name & $A$ & $A^{\mathcal{I}}(d) \in[0,1]$ \\
\hline truth constant & $\bar{p}$ & $p$ \\
\hline conjunction & $C \sqcap D$ & $\min \left\{C^{\mathcal{I}}(d), D^{\mathcal{I}}(d)\right\}$ \\
\hline implication & $C \rightarrow D$ & $C^{\mathcal{I}}(d) \Rightarrow D^{\mathcal{I}}(d)$ \\
\hline negation & $\neg C$ & $1-C^{\mathcal{I}}(d)$ \\
\hline existential restriction & $\exists r . C$ & $\sup _{e \in \Delta \mathcal{I}} \min \left\{r^{\mathcal{I}}(d, e), C^{\mathcal{I}}(e)\right\}$ \\
\hline value restriction & $\forall r . C$ & $\inf _{e \in \Delta^{\mathcal{I}}} r^{\mathcal{I}}(d, e) \Rightarrow C^{\mathcal{I}}(e)$ \\
\hline nominal & $\{a\}$ & $\begin{cases}1 & \text { if } d=a^{\mathcal{I}} \\
0 & \text { otherwise }\end{cases}$ \\
\hline at-least restriction & $\geqslant n s . C$ & $\sup _{\substack{e_{1}, \ldots, e_{n} \in \Delta^{\mathcal{I}} \\
\text { pairwise different }}} \min _{i=1}^{n} \min \left\{s^{\mathcal{I}}\left(d, e_{i}\right), C^{\mathcal{I}}\left(e_{i}\right)\right\}$ \\
\hline local reflexivity & $\exists s$.Self & $r^{\mathcal{I}}(d, d)$ \\
\hline role name & $r$ & $r^{\mathcal{I}}(d, e) \in[0,1]$ \\
\hline inverse role & $r^{-}$ & $r^{\mathcal{I}}(e, d)$ \\
\hline universal role & $r_{u}$ & 1 \\
\hline
\end{tabular}

$e, e^{\prime}, e_{1}, \ldots, e_{n} \in \Delta^{\mathcal{I}}$ such that $e_{1}, \ldots, e_{n}$ are pairwise different,

$$
\begin{aligned}
(\exists r . C)^{\mathcal{I}}(d) & =\min \left\{r^{\mathcal{I}}(d, e), C^{\mathcal{I}}(e)\right\}, \\
(\forall r . C)^{\mathcal{I}}(d) & =r^{\mathcal{I}}\left(d, e^{\prime}\right) \Rightarrow C^{\mathcal{I}}\left(e^{\prime}\right), \text { and } \\
(\geqslant n s . C)^{\mathcal{I}}(d) & =\min _{i=1}^{n} \min \left\{s^{\mathcal{I}}\left(d, e_{i}\right), C^{\mathcal{I}}\left(e_{i}\right)\right\} .
\end{aligned}
$$

As we have seen already in the role inclusions, the axioms of G-SROIQ extend classical axioms by stating a degree in $(0,1]$ to which the axioms hold. Moreover, we can compare the degrees of arbitrary classical assertions of the form $a: C$ or $(a, b): r$ for $a, b \in \mathrm{N}_{\mathrm{l}}, r \in \mathrm{N}_{\mathrm{R}}$, and a concept $C$. An order assertion [12] is of the form $\langle\alpha \bowtie p\rangle$ or $\langle\alpha \bowtie \beta\rangle$ for classical assertions $\alpha, \beta, \bowtie \in\{<, \leqslant,=, \geqslant,>\}$, and $p \in[0,1]$. An ordered ABox is a finite set of order assertions and individual (in)equality assertions of the form $a \approx b(a \not b)$ for $a, b \in \mathrm{N}_{\mathrm{I}}$. A general concept inclusion (GCI) is of the form $\langle C \sqsubseteq D \geqslant p\rangle$ for concepts $C, D$ and $p \in(0,1]$. A TBox is a finite set of GCIs. A disjoint role axiom is of the form $\langle\operatorname{dis}(r, s) \geqslant p\rangle$ for two simple roles $r, s \in \mathrm{N}_{\mathrm{R}}^{-}$and $p \in(0,1]$. A reflexivity axiom is of the form $\langle\operatorname{ref}(r) \geqslant p\rangle$ for a role $r \in \mathrm{N}_{\mathrm{R}}^{-}$and $p \in(0,1]$. An $R B$ ox $\mathcal{R}=\mathcal{R}_{h} \cup \mathcal{R}_{a}$ consists of a role hierarchy $\mathcal{R}_{h}$ and a finite set $\mathcal{R}_{a}$ of disjoint role and reflexivity axioms. An ontology $\mathcal{O}=(\mathcal{A}, \mathcal{T}, \mathcal{R})$ consists of an $\mathrm{ABox} \mathcal{A}$, a TBox $\mathcal{T}$, and an RBox $\mathcal{R}$.

A G-interpretation $\mathcal{I}$ satisfies (or is a model of) 
- an order assertion $\langle\alpha \bowtie \beta\rangle$ if $\alpha^{\mathcal{I}} \bowtie \beta^{\mathcal{I}}$ (where $p^{\mathcal{I}}:=p,(a: C)^{\mathcal{I}}:=C^{\mathcal{I}}\left(a^{\mathcal{I}}\right)$, and $\left.((a, b): r)^{\mathcal{I}}:=r^{\mathcal{I}}\left(a^{\mathcal{I}}, b^{\mathcal{I}}\right)\right)$;

- an (in)equality assertion $a \approx b(a \not b)$ if $a^{\mathcal{I}}=b^{\mathcal{I}}\left(a^{\mathcal{I}} \neq b^{\mathcal{I}}\right)$;

- a GCI $\langle C \sqsubseteq D \geqslant p\rangle$ if $C^{\mathcal{I}}(d) \Rightarrow D^{\mathcal{I}}(d) \geqslant p$ holds for all $d \in \Delta^{\mathcal{I}}$;

- a role inclusion $\left\langle r_{1} \ldots r_{n} \sqsubseteq r \geqslant p\right\rangle$ if $\left(r_{1} \ldots r_{n}\right)^{\mathcal{I}}\left(d_{0}, d_{n}\right) \Rightarrow r^{\mathcal{I}}\left(d_{0}, d_{n}\right) \geqslant p$ holds for all $d_{0}, d_{n} \in \Delta^{\mathcal{I}}$, where

$$
\left(r_{1} \ldots r_{n}\right)^{\mathcal{I}}\left(d_{0}, d_{n}\right):=\sup _{d_{1}, \ldots, d_{n-1} \in \Delta^{\mathcal{I}}} \min _{i=1}^{n} r_{i}^{\mathcal{I}}\left(d_{i-1}, d_{i}\right) ;
$$

- a disjoint role axiom $\langle\operatorname{dis}(r, s) \geqslant p\rangle$ if $\min \left\{r^{\mathcal{I}}(d, e), s^{\mathcal{I}}(d, e)\right\} \leqslant 1-p$ holds for all $d, e \in \Delta^{\mathcal{I}}$;

- a reflexivity axiom $\langle\operatorname{ref}(r) \geqslant p\rangle$ if $r^{\mathcal{I}}(d, d) \geqslant p$ holds for all $d \in \Delta^{\mathcal{I}}$;

- an ontology if it satisfies all its axioms.

An ontology is consistent if it has a (witnessed) model.

We can simulate other common role axioms in $\mathrm{G}-\mathcal{S R O I Q}[7,22]$ by those we introduced above:

- transitivity axioms $\langle\operatorname{tra}(r) \geqslant p\rangle$ by $\langle r r \sqsubseteq r \geqslant p\rangle$;

- symmetry axioms $\langle\operatorname{sym}(r) \geqslant p\rangle$ by $\left\langle r^{-} \sqsubseteq r \geqslant p\right\rangle$;

- asymmetry axioms $\langle\operatorname{asy}(s) \geqslant p\rangle$ by $\left\langle\operatorname{dis}\left(s, s^{-}\right) \geqslant p\right\rangle$;

- irreflexivity axioms $\langle\operatorname{irr}(s) \geqslant p\rangle$ by $\langle\exists s$. Self $\sqsubseteq \neg \bar{p} \geqslant 1\rangle$; and

- negated role assertions $\langle(a, b): \neg r \geqslant p\rangle$ by $\langle(a, b): r \leqslant 1-p\rangle$.

For an ontology $\mathcal{O}$, we denote by $\operatorname{rol}(\mathcal{O})$ the set of all roles occurring in $\mathcal{O}$, together with their inverses; by ind $(\mathcal{O})$ the set of all individual names occurring in $\mathcal{O}$, and by $\operatorname{sub}(\mathcal{O})$ the closure under negation of the set of all subconcepts occurring in $\mathcal{O}$. We consider $\neg \neg C$ to be equal to $C$, and thus $\operatorname{sub}(\mathcal{O})$ is of quadratic size in the size of $\mathcal{O}$. We denote by $\mathcal{V}_{\mathcal{O}}$ the closure under the involutive negation $x \mapsto 1-x$ of the set of all truth degrees appearing in $\mathcal{O}$ (either in axioms or in truth constants), together with $0,0.5$, and 1 . This set is of linear size.

Other common reasoning problems for FDLs, such as concept satisfiability and subsumption can be reduced to consistency [12]: the subsumption between $C$ and $D$ to degree $q$ w.r.t. a TBox $\mathcal{T}$ and an RBox $\mathcal{R}$ is equivalent to the inconsistency of $(\{\langle a: C \rightarrow D<q\rangle\}, \mathcal{T}, \mathcal{R})$, and the satisfiability of $C$ to degree $q$ w.r.t. $\mathcal{T}$ and $\mathcal{R}$ is equivalent to the consistency of $(\{\langle a: C \geqslant q\rangle\}, \mathcal{T}, \mathcal{R})$.

The letter $\mathcal{I}$ in G-SROIQ denotes the presence of inverse roles and the universal role. If such roles are not allowed, the resulting logic is written as $\mathrm{G}-\mathcal{S R O Q}$. Likewise, the name G-SRIQ indicates the absence of nominals, and G-SROI that of at-least and at-most restrictions. Replacing the letter $\mathcal{R}$ with $\mathcal{H}$ indicates that RBoxes are restricted to simple role inclusions, ABoxes are restricted to order assertions, and local reflexivity is not allowed; however, the letter $\mathcal{S}$ indicates that transitivity axioms are still allowed. Hence, in G-SHOIQ we can use role inclusions of the forms $\langle r \sqsubseteq s \geqslant p\rangle$ and $\langle r r \sqsubseteq r \geqslant p\rangle$. Disallowing axioms of the first type removes the letter $\mathcal{H}$, while the absence of transitivity axioms is denoted by replacing $\mathcal{S}$ with $\mathcal{A L C}$. 
Classical DLs are obtained from the above definitions by restricting the set of truth values to 0 and 1 . The semantics of a classical concept $C$ is then viewed as a set $C^{\mathcal{I}} \subseteq \Delta^{\mathcal{I}}$ instead of the characteristic function $C^{\mathcal{I}}: \Delta^{\mathcal{I}} \rightarrow\{0,1\}$, and likewise for roles. In this setting, all axioms (also order assertions) are restricted to be of the form $\langle\alpha \geqslant 1\rangle$, and usually this is simply written as $\alpha$, e.g. $C \sqsubseteq D$ instead of $\langle C \sqsubseteq D \geqslant 1\rangle$. We also use $C \equiv D$ to abbreviate $C \sqsubseteq D$ and $D \sqsubseteq C$. Furthermore, the implication constructor $C \rightarrow D$, although usually not included in classical DLs, can be expressed via $\neg C \sqcup D$.

In this paper, we provide a reduction from a G-SROIQ ontology to a classical $\mathcal{A L C O Q}$ ontology. For all sublogics of $\mathrm{G}-\mathcal{S} \mathcal{R O} \mathcal{I} \mathcal{Q}$ that do not contain the constructors $\mathcal{O}, \mathcal{I}$, and $\mathcal{Q}$ at the same time, the reduction preserves consistency. Before we describe the main reduction, however, we provide a characterization of role hierarchies using (weighted) finite automata.

\section{Automata for Complex Role Inclusions}

Let $\mathcal{O}=(\mathcal{A}, \mathcal{T}, \mathcal{R})$ be a $\mathrm{G}-\mathcal{S} \mathcal{R O} \mathcal{O} \mathcal{Q}$ ontology. We extend the idea from [23] of using finite automata to characterize all role chains that imply a given role w.r.t. $\mathcal{R}_{h}$. Here, we use a kind of weighted automata [19], which use as input symbols the roles in $\operatorname{rol}(\mathcal{O})$, and compute a weight for any given input word.

Definition 1 (WFA). A weighted finite automaton (WFA) is a quadruple $\mathbf{A}=\left(Q, q_{\mathrm{ini}}, \mathrm{wt}, q_{\mathrm{fin}}\right)$, consisting of a non-empty set $Q$ of states, an initial state $q_{\text {ini }} \in Q, a$ transition weight function wt: $Q \times(\operatorname{rol}(\mathcal{O}) \cup\{\varepsilon\}) \times Q \rightarrow[0,1]$, and a final state $q_{\text {fin }} \in Q$. Given an input word $w \in \operatorname{rol}(\mathcal{O})^{*}$, a run of $\mathbf{A}$ on $w$ is a non-empty sequence of pairs $\mathbf{r}=\left(w_{i}, q_{i}\right)_{0 \leqslant i \leqslant m}$ such that $\left(w_{0}, q_{0}\right)=\left(w, q_{\text {ini }}\right)$, $\left(w_{m}, q_{m}\right)=\left(\varepsilon, q_{\text {fin }}\right)$, and for each $i, 1 \leqslant i \leqslant m$, it holds that $w_{i-1}=x_{i} w_{i}$ for some $x_{i} \in \operatorname{rol}(\mathcal{O}) \cup\{\varepsilon\}$. The weight of such a run is $\operatorname{wt}(\mathrm{r}):=\min _{i=1}^{m} \operatorname{wt}\left(q_{i-1}, x_{i}, q_{i}\right)$. The behavior of $\mathbf{A}$ on $w$ is $(\|\mathbf{A}\|, w):=\sup _{\mathbf{r} \text { run of } \mathbf{A} \text { on } w} \operatorname{wt}(\mathbf{r})$.

We often denote by $q \stackrel{x, p}{\longrightarrow} q^{\prime} \in \mathbf{A}$ the fact that $\operatorname{wt}\left(q, x, q^{\prime}\right)=p$. Further, for a state $q$ of $\mathbf{A}$, we denote by $\mathbf{A}^{q}$ the automaton resulting from $\mathbf{A}$ by making $q$ the initial state.

Following [23], we now construct, for each role $r$, a WFA $\mathbf{A}_{r}$ that recognizes all role chains that "imply" $r$ w.r.t. $\mathcal{R}_{h}$ (with associated degrees). This construction proceeds in several steps. The first automaton $\mathbf{A}_{r}^{0}$ contains the initial state $i_{r}$, the final state $f_{r}$, and the transition $i_{r} \stackrel{r, 1}{\longrightarrow} f_{r}$, as well as the following transitions for each $\langle w \sqsubseteq r \geqslant p\rangle \in \mathcal{R}$ :

- if $w=r r$, then $f_{r} \stackrel{\varepsilon, p}{\longrightarrow} i_{r}$;

- if $w=r_{1} \ldots r_{n}$ with $r_{1} \neq r \neq r_{n}$, then $i_{r} \stackrel{r_{1}, 1}{\longrightarrow} q_{w}^{1} \stackrel{r_{2}, 1}{\longrightarrow} \ldots \stackrel{r_{n}, 1}{\longrightarrow} q_{w}^{n} \stackrel{\varepsilon, p}{\longrightarrow} f_{r}$;

- if $w=r r_{1} \ldots r_{n}$, then $f_{r} \stackrel{r_{1}, 1}{\longrightarrow} q_{w}^{1} \stackrel{r_{2}, 1}{\longrightarrow} \ldots \stackrel{r_{n}, 1}{\longrightarrow} q_{w}^{n} \stackrel{\varepsilon, p}{\longrightarrow} f_{r}$; and

- if $w=r_{1} \ldots r_{n} r$, then $i_{r} \stackrel{r_{1}, 1}{\longrightarrow} q_{w}^{1} \stackrel{r_{2}, 1}{\longrightarrow} \ldots \stackrel{r_{n}, 1}{\longrightarrow} q_{w}^{n} \stackrel{\varepsilon, p}{\longrightarrow} i_{r}$, 
where all states $q_{w}^{i}$ are distinct. Here and in the following, all transitions that are not explicitly mentioned have weight 0 .

The WFA $\mathbf{A}_{r}^{1}$ is now defined as $\mathbf{A}_{r}^{0}$ if there is no role inclusion of the form $\left\langle r^{-} \sqsubseteq r \geqslant p\right\rangle \in \mathcal{R}$; otherwise, $\mathbf{A}_{r}^{1}$ is the disjoint union of $\mathbf{A}_{r}^{0}$ and a mirrored copy of $\mathbf{A}_{r}^{0}$, where $i_{r}$ is the only initial state, $f_{r}$ is the only final state, and the following transitions are added for the copy $f_{r}^{\prime}$ of $f_{r}$ and the copy $i_{r}^{\prime}$ of $i_{r}$ : $i_{r} \stackrel{\varepsilon, p}{\longrightarrow} f_{r}^{\prime}, f_{r}^{\prime} \stackrel{\varepsilon, p}{\longrightarrow} i_{r}, f_{r} \stackrel{\varepsilon, p}{\longrightarrow} i_{r}^{\prime}$, and $i_{r}^{\prime} \stackrel{\varepsilon, p}{\longrightarrow} f_{r}$.

Finally, we define the WFA $\mathbf{A}_{r}$ by induction on $\prec$ as follows:

- if $r$ is minimal w.r.t. $\prec$, then $\mathbf{A}_{r}:=\mathbf{A}_{r}^{1}$;

- otherwise, $\mathbf{A}_{r}$ is the disjoint union of $\mathbf{A}_{r}^{1}$ with a copy $\mathbf{A}_{s}^{1^{\prime}}$ of $\mathbf{A}_{s}^{1}$ for each transition $q \stackrel{s, 1}{\longrightarrow} q^{\prime}$ in $\mathbf{A}_{r}^{1}$ with $s \neq r .{ }^{7}$ For each such transition, we add $\varepsilon$-transitions with weight 1 from $q$ to the initial state of $\mathbf{A}_{s}^{1^{\prime}}$ and from the final state of $\mathbf{A}_{s}^{1^{\prime}}$ to $q^{\prime}$.

- The automaton $\mathbf{A}_{r-}$ is a mirrored copy of $\mathbf{A}_{r}$.

The difference to the construction in [23] is only the inclusion of the appropriate weights for each considered role inclusion. As shown in [23], the size of each $\mathbf{A}_{r}$ is bounded exponentially in the length of the longest chain $r_{1} \prec \cdots \prec r_{n}$ for which there are role inclusions $\left\langle u_{i} r_{i-1} v_{i} \sqsubseteq r_{i} \geqslant p_{i}\right\rangle \in \mathcal{R}$ for all $i, 2 \leqslant i \leqslant n$.

The following generalization of [23, Proposition 9] describes the promised characterization of the role inclusions in $\mathcal{R}$ in terms of the behavior of the automata $\mathbf{A}_{r}$. A detailed proof can be found in [16].

Lemma 2. A G-interpretation $\mathcal{I}$ satisfies all role inclusions in $\mathcal{R}$ iff for every $r \in \operatorname{rol}(\mathcal{O})$, every $w \in \operatorname{rol}(\mathcal{O})^{+}$, and all $d, e \in \Delta^{\mathcal{I}}$, we have

$$
w^{\mathcal{I}}(d, e) \Rightarrow r^{\mathcal{I}}(d, e) \geqslant\left(\left\|\mathbf{A}_{r}\right\|, w\right)
$$

Intuitively, the degree to which the interpretation of $w$ must be "included" in the interpretation of $r$ is determined by the behavior of $\left\|\mathbf{A}_{r}\right\|$ on $w$.

For the universal role $r_{u}$, we define $\mathbf{A}_{r_{u}}$ as above based on the role inclusions $\left\langle r_{u}^{-} \sqsubseteq r_{u} \geqslant 1\right\rangle,\left\langle r_{u} r_{u} \sqsubseteq r_{u} \geqslant 1\right\rangle$, and $\left\langle r \sqsubseteq r_{u} \geqslant 1\right\rangle$ for all $r \in \operatorname{rol}(\mathcal{O})$. Hence, $\mathbf{A}_{r_{u}}$ accepts any (non-empty) word $w \in \operatorname{rol}(\mathcal{O})^{+}$with degree 1 , and it is easy to see that Lemma 2 also holds for $r_{u}$.

\section{The Reduction}

We now describe the reduction from $\mathcal{O}$ to a classical $\mathcal{A L C O} \mathcal{Q}$ ontology $\operatorname{red}(\mathcal{O})$. This reduction always uses nominals, even in the logic G-SRIQ . However, if number restrictions are not allowed (e.g. in $\mathrm{G}-\mathcal{S R O} \mathcal{I}$ ), then $\operatorname{red}(\mathcal{O})$ is an $\mathcal{A L C O}$ ontology. For ease of presentation, we consider here only the FDL G-SROQ without (local) reflexivity statements of the form $\exists r$.Self or $\langle\operatorname{ref}(r) \geqslant p\rangle$. In the presence of these constructors and inverse roles, the reduction contains some

\footnotetext{
$\overline{7}$ Note that all transitions labeled with roles have weight 0 or 1.
} 
additional concepts and axioms, but the main ideas remain the same. The full construction can be found in [16].

We first extend the $\operatorname{set} \operatorname{sub}(\mathcal{O})$ by all nominals $\{a\}, a \in \operatorname{ind}(\mathcal{O})$, (and their negations) to be able to distinguish all named domain elements. We further add all "concepts" of the form $\forall \mathbf{A}_{r}^{q} . C\left(\exists \mathbf{A}_{r}^{q} . C\right)$ for all $\forall r . C(\exists r . C)$ occurring in $\mathcal{O}$ and all states $q$ of $\mathbf{A}_{r}$. These concepts help to transfer the constraints imposed by the existential and value restrictions along all role chains that imply the possibly non-simple role $r$. The semantics of $\forall \mathbf{A} . C$ is defined as follows:

$$
(\forall \mathbf{A} . C)^{\mathcal{I}}(d):=\inf _{w \in \operatorname{rol}(\mathcal{O})^{*}} \inf _{e \in \Delta^{\mathcal{I}}} \min \left\{(\|\mathbf{A}\|, w), w^{\mathcal{I}}(d, e)\right\} \Rightarrow C^{\mathcal{I}}(e),
$$

where $\varepsilon^{\mathcal{I}}(d, e):=1$ if $d=e$, and $\varepsilon^{\mathcal{I}}(d, e):=0$ otherwise. Intuitively, it behaves like a value restriction, but instead of considering only the role $r$, we consider any role chain $w$, weighted by the behavior of $\mathbf{A}$ on $w$. Recall that for $\mathbf{A}_{r}$, this behavior represents the degree to which $w$ implies $r$ w.r.t. $\mathcal{R}_{h}$ (see Lemma 2).

The idea is that in our reduction we do not need to explicitly represent all role connections, but only a "skeleton" of connections which are necessary to satisfy the witnessing conditions for role restrictions. The restrictions for all implied role connections are then handled by the concepts $\forall \mathbf{A}_{r} . C$ and $\exists \mathbf{A}_{r} . C$ by simulating the transitions of $\mathbf{A}_{r}$; each transition corresponds to a role connection to a new domain element. Note that we do not need to introduce concepts of the form $\geqslant n \mathbf{A}_{r} . C$ since all roles in at-least restrictions must be simple, i.e. there can be no role chains of length $>1$ that imply them (at least not with a degree $>0$ ).

The main idea of the reduction is that instead of precisely defining the interpretation of all concepts at each domain element, it suffices to consider a total preorder on them. For example, if an axiom restricts the value of $C \rightarrow D$ at each domain element to be $\geqslant 0.5$, then we do not have to find the exact values of $C$ and $D$, but only to ensure that either $C^{\mathcal{I}}(d) \leqslant D^{\mathcal{I}}(d)$ or else $D^{\mathcal{I}}(d) \geqslant 0.5$. This information is encoded by total preorders over the order structure $\mathcal{U}$ that is defined below. The other main insight for our reduction is that we consider only (quasi-)forest-shaped models of $\mathcal{O}$ [17]. In such a model, the domain elements identified by individual names serve as the roots of several tree-shaped structures. The roots themselves may be arbitrarily interconnected by roles. Due to nominals, there may also be role connections from any domain element back to the roots. Note that complex role inclusions may actually imply role connections between arbitrary domain elements, but the underlying tree-shaped "skeleton" is what is important for reasoning purposes (for details, see [17] and our correctness proof in [16]). This dependence on forest-shaped models is the reason why our reduction works only for $\mathrm{G}-\mathcal{S} \mathcal{R O} \mathcal{I}, \mathrm{G}-\mathcal{S} \mathcal{R O Q}$, and $\mathrm{G}-\mathcal{S} \mathcal{R} \mathcal{I} \mathcal{Q}$ - even classical

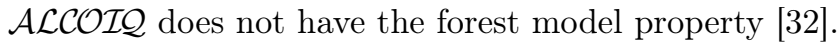

We now define the order structure $\mathcal{U}$ as follows:

$$
\begin{aligned}
& \mathcal{U}_{\mathcal{A}}:=\mathcal{V}_{\mathcal{O}} \cup\{a: C \mid a \in \operatorname{ind}(\mathcal{O}), C \in \operatorname{sub}(\mathcal{O})\} \cup \\
&\{(a, b): s \mid a, b \in \operatorname{ind}(\mathcal{O}), r \in \operatorname{rol}(\mathcal{O}), s \in\{r, \neg r\}\} \\
& \mathcal{U}:=\mathcal{U}_{\mathcal{A}} \cup \operatorname{sub}(\mathcal{O}) \cup \operatorname{sub}_{\uparrow}(\mathcal{O}) \cup \\
&\{s,(*, a): s \mid a \in \operatorname{ind}(\mathcal{O}), r \in \operatorname{rol}(\mathcal{O}), s \in\{r, \neg r\}\},
\end{aligned}
$$


where $\operatorname{sub}_{\uparrow}(\mathcal{O}):=\left\{\langle C\rangle_{\uparrow} \mid C \in \operatorname{sub}(\mathcal{O})\right\}$ and the function inv is defined by $\operatorname{inv}(C):=\neg C, \operatorname{inv}(a: C):=a: \neg C, \operatorname{inv}(*, a): r:=(*, a): \neg r$, etc.

Total preorders on assertions in $\mathcal{U}_{\mathcal{A}}$ are used to describe the behavior of the named root elements in the forest-shaped model. For example, if the order is such that $a: C>(a, b): r$, the idea is that in the corresponding $\mathrm{G}$-model $\mathcal{I}$ of $\mathcal{O}$ the value of $C$ at $a$ is strictly greater that the value of the $r$-connection from $a$ to $b$, i.e. $C^{\mathcal{I}}\left(a^{\mathcal{I}}\right)>r^{\mathcal{I}}\left(a^{\mathcal{I}}, b^{\mathcal{I}}\right)$. For each domain element of $\mathcal{I}$, total preorders on the elements of $\operatorname{sub}(\mathcal{O})$ describe the degrees of all relevant concepts in a similar way. The elements of $\operatorname{sub}_{\uparrow}(\mathcal{O})$ are used to refer to degrees of concepts at the unique predecessor element in the tree-shaped parts of the interpretation. For convenience, we also define $\langle p\rangle_{\uparrow}:=p$ for all $p \in \mathcal{V}_{\mathcal{O}}$. The elements $r \in \operatorname{rol}(\mathcal{O})$ represent the values of the role connections from the predecessor. The special elements $(*, a): r$ describe role connections between arbitrary domain elements (represented by $*$ ) and the named elements in the roots.

In order to describe total preorders over $\mathcal{U}$ with a classical $\mathcal{A L C O Q}$ ontology, we use special concept names of the form $\alpha \leqslant \beta$ for $\alpha, \beta \in \mathcal{U}$. This differs from previous reductions for finitely valued FDLs $[7,9,31]$ in that we not only consider cut-concepts of the form $p \leqslant C$ with $p \in \mathcal{V}_{\mathcal{O}}$, but also relationships between different concepts. ${ }^{8}$ We use the abbreviations $\alpha \geqslant \beta:=\beta \leqslant \alpha, \alpha<\beta:=-\alpha \geqslant \beta$, and similarly for $=$ and $>$. Furthermore, we define the complex expressions

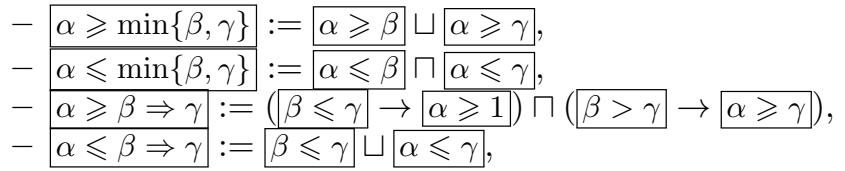

and extend these notions to $\alpha \bowtie \beta \Rightarrow \gamma$ etc., for $\bowtie \in\{<,=,>\}$, analogously.

In our reduction, we additionally use the special concept name AN to identify the anonymous domain elements, i.e. those which are not of the form $b^{\mathcal{I}}$ for any $b \in \operatorname{ind}(\mathcal{O})$. The reduction uses only one classical role name $\mathfrak{r}$, which simulates the tree structure of the fuzzy interpretation; the actual values of the fuzzy roles in this tree are expressed using the elements in $\mathcal{U}$. The reduced ontology red $(\mathcal{O})$ consists of the parts $\operatorname{red}(\mathcal{U}), \operatorname{red}(\mathcal{A}), \operatorname{red}(\mathrm{AN}), \operatorname{red}(\uparrow), \operatorname{red}(\mathcal{R}), \operatorname{red}(\mathcal{T})$, and $\operatorname{red}(C)$ for all $C \in \operatorname{sub}(\mathcal{O})$, which we define in the following. We emphasize again that $\operatorname{red}(\mathcal{O})$ is formulated in $\mathcal{A L C O Q}$, whenever $\mathcal{O}$ is in G-SRIQ or G-SROQ , and in $\mathcal{A L C O}$ if $\mathcal{O}$ is a $\mathrm{G}-\mathcal{S R O I}$ ontology. This is due to the fact that we always use nominals to distinguish the named from the anonymous part of the forest-shaped model, and the inverse of $\mathfrak{r}$ is not needed in the reduction (see [16] for details).

The first part of $\operatorname{red}(\mathcal{O})$ is

$$
\begin{aligned}
\operatorname{red}(\mathcal{U}):= & \{\alpha \leqslant \beta \sqcap \beta \leqslant \gamma \sqsubseteq \alpha \leqslant \gamma \mid \alpha, \beta, \gamma \in \mathcal{U}\} \cup \\
& \{T \sqsubseteq \alpha \leqslant \beta \sqcup \beta \leqslant \alpha \mid \alpha, \beta \in \mathcal{U}\} \cup \\
& \{T \sqsubseteq 0 \leqslant \alpha \sqcap \alpha \leqslant 1 \mid \alpha \in \mathcal{U}\} \cup \\
& \left\{T \sqsubseteq \alpha \bowtie \beta \mid \alpha, \beta \in \mathcal{V}_{\mathcal{O}}, \alpha \bowtie \beta\right\} \cup \\
& \{\alpha \leqslant \beta \sqsubseteq \operatorname{inv}(\beta) \leqslant \operatorname{inv}(\alpha) \mid \alpha, \beta \in \mathcal{U}\} .
\end{aligned}
$$

\footnotetext{
$\overline{{ }^{8} \text { For the rest }}$ of this paper, the expressions $\alpha \leqslant \beta$ denote classical concept names.
} 
These axioms ensure that at each domain element the relation " $\leqslant$ " forms a total preorder compatible with the values in $\mathcal{V}_{\mathcal{O}}$, and that inv is an antitone operator.

To describe the behavior of the named elements, we use the following axioms:

$$
\begin{aligned}
\operatorname{red}(\mathcal{A}):= & \{c: \alpha \bowtie \beta \mid\langle\alpha \bowtie \beta\rangle \in \mathcal{A}\} \cup\{a \approx b \in \mathcal{A}\} \cup\{a \not b \in \mathcal{A}\} \cup \\
& \{(a, b): \mathfrak{r} \mid a, b \in \operatorname{ind}(\mathcal{O})\} \cup\left\{\alpha \bowtie \beta \sqsubseteq \forall \mathfrak{r} . \alpha \bowtie \beta \mid \alpha, \beta \in \mathcal{U}_{\mathcal{A}}\right\} \cup \\
& \{a: a: C=C \mid a \in \operatorname{ind}(\mathcal{O}), C \in \operatorname{sub}(\mathcal{O})\} \cup \\
& \{a:(a, b): r=(*, b): r \mid a, b \in \operatorname{ind}(\mathcal{O}), r \in \operatorname{rol}(\mathcal{O})\},
\end{aligned}
$$

where $c$ is an arbitrary individual name. The first two lines are responsible for enforcing that the ABox is satisfied and that information about the behavior of the named individuals is available throughout the whole model. The remaining axioms describe various equivalences for named individuals, e.g. that $(a, b): r$ and $(*, b): r$ should have the same value when evaluated at $a$.

The next axiom defines the concept $\mathrm{AN}$ of all anonymous elements, i.e. those that are not designated by an individual name:

$$
\operatorname{red}(\mathrm{AN}):=\left\{\neg \mathrm{AN} \equiv \bigsqcup_{a \in \operatorname{ind}(\mathcal{O})}\{a\}\right\}
$$

The following axioms ensure that the order of an element in a tree-shaped part of the model is known at each of its successors via the elements of $\operatorname{sub}_{\uparrow}(\mathcal{O})$ :

$$
\operatorname{red}(\uparrow):=\left\{\alpha \bowtie \beta \sqsubseteq \forall \mathfrak{r} .\left(\mathrm{AN} \rightarrow\langle\alpha\rangle_{\uparrow} \bowtie\langle\beta\rangle_{\uparrow}\right) \mid \alpha, \beta \in \mathcal{V}_{\mathcal{O}} \cup \operatorname{sub}(\mathcal{O})\right\}
$$

We now come to the reduction of the RBox:

$$
\begin{aligned}
& \operatorname{red}(\mathcal{R}):=\{\top \sqsubseteq(a, b): r \Rightarrow(a, b): s \geqslant p \sqcap r \Rightarrow s \geqslant p \\
& \langle r \sqsubseteq s \geqslant p\rangle \in \mathcal{R}, a, b \in \operatorname{ind}(\mathcal{O}) \cup\{*\}\} \cup \\
& \{\top \sqsubseteq \min \{(a, b): r,(a, b): s\} \leqslant 1-p \sqcap \min \{r, s\} \leqslant 1-p \mid \\
& \langle\operatorname{dis}(r, s) \geqslant p\rangle \in \mathcal{R}, a, b \in \operatorname{ind}(\mathcal{O}) \cup\{*\}\}
\end{aligned}
$$

These axioms ensure that the various elements of $\mathcal{U}$ that represent the values of role connections, such as $(a, b): r$ and $r$, respect the axioms in $\mathcal{R}$. Although simple role inclusions $\langle r \sqsubseteq s \geqslant p\rangle$ are handled by the automata $\mathbf{A}_{r}$, we include them also here. The reason is that the reduction of at-least restrictions below does not need to use these automata since only simple roles can occur in them.

The GCIs in $\mathcal{T}$ can be translated in a straightforward manner:

$$
\operatorname{red}(\mathcal{T}):=\{\top \sqsubseteq p \leqslant C \Rightarrow D \mid\langle C \sqsubseteq D \geqslant p\rangle \in \mathcal{T}\}
$$

We now come to the reductions of the concepts. Intuitively, each $\operatorname{red}(C)$ with $C \in \operatorname{sub}(\mathcal{O})$ describes the semantics of $C$ in terms of its order relationships to other elements of $\mathcal{U}$. Note that the semantics of the involutive negation 
$\neg C=\operatorname{inv}(C)$ is already handled by the operator inv ( $\operatorname{see} \operatorname{red}(\mathcal{U})$ above):

$$
\begin{aligned}
\operatorname{red}(\top) & :=\{\top \sqsubseteq \top \geqslant 1\} \\
\operatorname{red}(\{a\}) & :=\{\{a\} \sqsubseteq 1 \leqslant\{a\}, \neg\{a\} \sqsubseteq\{a\} \leqslant 0\} \\
\operatorname{red}(\bar{p}) & :=\{\top \sqsubseteq \bar{p}=p \mid\} \\
\operatorname{red}(\neg C) & :=\emptyset \\
\operatorname{red}(C \sqcap D) & :=\{\top \sqsubseteq C \sqcap D=\min \{C, D\} \\
\operatorname{red}(C \rightarrow D) & :=\{\top \sqsubseteq C \rightarrow D=C \Rightarrow D\}
\end{aligned}
$$

The reductions of role restrictions are more involved. In particular, in the case of value and existential restrictions we have to deal with non-simple roles, for which we employ the automata $\mathbf{A}_{r}$ from the previous section:

$$
\begin{aligned}
& \operatorname{red}(\forall r . C):=\left\{\top \sqsubseteq(\forall r . C) \leqslant\left(\forall \mathbf{A}_{r} \cdot C\right),\right. \\
& \top \sqsubseteq \exists \mathfrak{r} .\left(\mathrm{AN} \sqcap\langle\forall r . C\rangle_{\uparrow} \geqslant r \Rightarrow C\right) \sqcup \\
& \left.\bigsqcup_{a \in \operatorname{ind}(\mathcal{O})}(\exists \mathfrak{r} .\{a\} \sqcap(\forall r . C) \geqslant(*, a): r \Rightarrow a: C)\right\}
\end{aligned}
$$

The second axiom of $\operatorname{red}(\forall r . C)$ ensures the existence of a witness for $\forall r . C$ at each domain element. For example, assume that the preorder represented by the concepts $\alpha \leqslant \beta$ at some domain element $d$ satisfies $0.5<\forall$ r.C $<1$. The first possibility is that the above axiom creates an anonymous element $e$ that is connected to $d$ via $\mathfrak{r}$, and hence by red(AN) we know that $e$ satisfies $0.5<\langle\forall r . C\rangle_{\uparrow}<1$. The axiom further requires that $\langle\forall r . C\rangle_{\uparrow} \geqslant r \Rightarrow C$, which implies that $\langle\forall r . C\rangle_{\uparrow} \geqslant C$ and $r>C$. We will see below that the reduction of $\forall \mathbf{A}_{r} . C$ further ensures that $\langle\forall r . C\rangle_{\uparrow} \leqslant r \Rightarrow C$, and thus we get $\langle\forall r . C\rangle_{\uparrow}=C$. Hence, $e$ can be seen as an abstract representation of the witness of $\forall r . C$ at $d$; the precise value of the $r$ connection between $d$ and $e$ (represented by the element $r$ ) is irrelevant, as long as it is strictly greater than the value of $C$ at $e$. The other disjuncts of this axiom deal with the possibility that a named domain element acts as the witness for the value restriction in a similar way.

Together with the first axiom of $\operatorname{red}(\forall r . C)$, the following axioms ensure that no other $r$-successor of $d$ violates the lower bound on $r \Rightarrow C$ given by $\forall r . C$ at $d$ :

$$
\begin{aligned}
& \operatorname{red}\left(\forall \mathbf{A}^{q} . C\right):=\left\{\top \sqsubseteq\left(\forall \mathbf{A}^{q} . C\right) \leqslant C \mid q \text { is final }\right\} \cup \bigcup \operatorname{red}_{x, p, q^{\prime}}\left(\forall \mathbf{A}^{q} . C\right) \\
& q \stackrel{x, p}{\longrightarrow} q^{\prime} \in \mathbf{A} \\
& \operatorname{red}_{\varepsilon, p, q^{\prime}}\left(\forall \mathbf{A}^{q} . C\right):=\left\{\top \sqsubseteq\left(\forall \mathbf{A}^{q} . C\right) \leqslant p \Rightarrow\left(\forall \mathbf{A}^{q^{\prime}} . C\right)\right\} \\
& \operatorname{red}_{s, p, q^{\prime}}\left(\forall \mathbf{A}^{q} . C\right):= \\
& \left\{\top \sqsubseteq \forall \mathfrak{r} .\left(\mathrm{AN} \rightarrow\left\langle\forall \mathbf{A}^{q} . C\right\rangle_{\uparrow} \leqslant \min \{p, s\} \Rightarrow\left(\forall \mathbf{A}^{q^{\prime}} . C\right)\right)\right\} \cup \\
& \left\{\exists \mathfrak{r} .\{a\} \sqsubseteq\left(\forall \mathbf{A}^{q} . C\right) \leqslant \min \{p,(*, a): s\} \Rightarrow a:\left(\forall \mathbf{A}^{q^{\prime}} . C\right) \mid a \in \operatorname{ind}(\mathcal{O})\right\}
\end{aligned}
$$

Recall that $\mathbf{A}_{r}$ in particular contains the transition $i_{r} \stackrel{r, 1}{\longrightarrow} f_{r}$ from the initial state $i_{r}$ to the final state $f_{r}$. By the first axiom in $\operatorname{red}(\forall r . C)$ and the first axiom 
in $\operatorname{red}_{r, 1, f_{r}}\left(\forall \mathbf{A}_{r} . C\right)$, the witness $e$ satisfies $\langle\forall r . C\rangle_{\uparrow} \leqslant\left\langle\forall \mathbf{A}_{r} . C\right\rangle_{\uparrow} \leqslant r \Rightarrow\left(\forall \mathbf{A}_{r}^{f_{r}} . C\right)$ Since $f_{r}$ is final, we further have $\left(\forall \mathbf{A}_{r}^{f_{r}} . C\right) \leqslant C$ by $\operatorname{red}\left(\forall \mathbf{A}_{r}^{f_{r}} . C\right)$, and hence $\langle\forall r . C\rangle_{\uparrow} \leqslant r \Rightarrow C$, as claimed above.

Using arbitrary runs through the automaton $\mathbf{A}_{r}$, we can ensure that no other $r$-successor of $d$ violates the value restriction. For example, if $r^{\mathcal{I}}\left(d, e_{1}\right)=0.3$ and $r^{\mathcal{I}}\left(e_{1}, e_{2}\right)=0.5$ for two other (anonymous) domain elements $e_{1}, e_{2}$, and we further have the role inclusion $\langle r r \sqsubseteq r \geqslant 0.7\rangle$, then we know that $r^{\mathcal{I}}\left(d, e_{2}\right)$ must be at least 0.5 . Although this $r$-connection is not explicitly represented in our forest-based encoding, concepts of the form $\forall \mathbf{A}_{r}^{q} . C$ are appropriately transferred from $d$ via $e_{1}$ to $e_{2}$ in order to ensure that the value of $C$ at $e_{2}$ satisfies $0.5<(\forall r . C)^{\mathcal{I}}(d) \leqslant r^{\mathcal{I}}\left(d, e_{2}\right) \Rightarrow C^{\mathcal{I}}\left(e_{2}\right)$. In this example, since we know only that $r^{\mathcal{I}}\left(d, e_{2}\right) \geqslant 0.5$, it must be ensured that $C^{\mathcal{I}}\left(e_{2}\right) \geqslant r^{\mathcal{I}}\left(d, e_{2}\right)$.

The reduction for existential restrictions can be defined similarly to that for value restrictions, but replacing $\geqslant$ with $\leqslant$ (and vice versa) and $\Rightarrow$ with $\min$.

We now come to the final component of $\operatorname{red}(\mathcal{O})$ :

$$
\begin{aligned}
& \operatorname{red}(\geqslant n r . C):=\left\{\top \sqsubseteq \bigsqcup_{m=0}^{n} \bigsqcup_{\substack{S \subseteq \operatorname{ind}(\mathcal{O}) \\
|S|=n-m}}\right\rceil \operatorname{red}_{m, S, \leqslant}(\geqslant n r . C) \text {, } \\
& \left.\mathrm{AN} \sqsubseteq \neg \bigsqcup_{m=0}^{n} \bigsqcup_{S \subseteq \text { ind }(\mathcal{O})} \sqcap \operatorname{red}_{m, S,<}(\geqslant n r . C)\right\} \cup \\
& |S|=n-m \\
& \left\{a: \neg \geqslant n \mathfrak{r} .\left(\left(\mathrm{AN} \sqcap\langle\geqslant n r . C\rangle_{\uparrow}<\min \{r, C\}\right) \sqcup\right.\right. \\
& (\neg \mathrm{AN} \sqcap(a: \geqslant n r . C)<\min \{(a, *): r, C\})) \mid a \in \operatorname{ind}(\mathcal{O})\} \\
& \operatorname{red}_{m, S, \triangleleft}(\geqslant n r . C):=\left\{\geqslant m \mathfrak{r} .\left(\text { AN } \sqcap\langle\geqslant n r . C\rangle_{\uparrow} \triangleleft \min \{r, C\}\right)\right\} \cup \\
& \{\exists \mathfrak{r} .(\{a\} \sqcap \neg\{b\}) \mid a, b \in S, a \neq b\} \cup \\
& \{((\geqslant n r . C) \triangleleft \min \{(*, a): r, a: C\} \mid a \in S\}
\end{aligned}
$$

The reduction of at-least restrictions works similarly to the one of value restrictions: the first axiom ensures the existence of the $n$ required witnesses, while the second one ensures that no $n$ different elements can exceed the value of the at-least restriction. Unfortunately, the number of named successors cannot be counted using a classical at-least restriction in our encoding, since these named successors do not know about the degree of the role connection from an anonymous element; otherwise they would have to store a possibly infinite amount of information since they may have infinitely many anonymous role predecessors. For this reason, the above axioms first guess how many $(n-m)$ and which $(S)$ named elements are connected to the current domain element to the appropriate degrees (given by $(*, a): r)$. The assertions in $\operatorname{red}(\geqslant n r . C)$ express a restriction similar to that of the second GCI for named domain elements.

The proof of the following correctness result can be found in [16]. As mentioned before, this holds only for logics with the forest model property [17]. However, it is not affected by the presence or absence of (local) reflexivity. 
Lemma 3. In $\mathrm{G}-\mathcal{S} \mathcal{R} \mathcal{I Q}, \mathrm{G}-\mathcal{S} \mathcal{R O Q}$, or $\mathrm{G}-\mathcal{S R O} \mathcal{I}$, $\mathcal{O}$ has a $\mathrm{G}$-model iff red $(\mathcal{O})$ has a classical model.

We now analyze the complexity of the reduction. As in [23], the construction of the automata $\mathbf{A}_{r}$ causes an exponential blowup in the size of $\mathcal{R}$, which cannot be avoided [26]. Independent of this, our reduction also involves an exponential blowup in the (binary encoding of) the largest number $n$ involved in a number restriction in $\mathcal{O}$, and in the number of individual names occurring in $\mathcal{O}$, since the number of disjuncts in each GCI from $\operatorname{red}(\geqslant n r . C)$ is linear in $n \cdot 2^{\mid \text {ind }(\mathcal{O}) \mid}$. However, we can avoid this blowup if we remove either nominals or number restrictions [16]. Hence, we obtain the following complexity results.

Theorem 4. Deciding consistency is

- 2-EXPTime-complete in G-SRIQ

- in 2-EXPTIME in G-SROI and G-SROQ , and

- ExpTime-complete in all FDLs between $\mathrm{G}-\mathcal{A L C}$ and $\mathrm{G}-\mathcal{S H O I}$ or $\mathrm{G}-\mathcal{S H I Q}$.

Proof. The consistency of the $\mathcal{A L C O Q}$ ontology red $(\mathcal{O})$ is decidable in exponential time in the size of $\operatorname{red}(\mathcal{O})$ [17]. The first upper bounds thus follow from the fact that the size of $\operatorname{red}(\mathcal{O})$ is exponential in the size of $\mathcal{O}$. 2-ExpTime-hardness holds already for $\mathrm{G}-\mathcal{S} \mathcal{R} \mathcal{I} \mathcal{Q}$ without involutive negation and only assertions of the form $\langle\alpha \geqslant p\rangle$ since in this case reasoning in $\mathrm{G}-\mathcal{S} \mathcal{R} \mathcal{I Q}$ is equivalent to reasoning in classical $\mathcal{S R I Q}[13,26]$.

Without complex role inclusions, i.e. restricting to simple role inclusions and transitivity axioms, the size of the automata $\mathbf{A}_{r}$ is polynomial in the size of $\mathcal{R}$ [23]. The other exponential blowup can be avoided by disallowing nominals or number restrictions. Hence, for G-SHOI and G-SHIQ, the size of $\operatorname{red}(\mathcal{O})$ is polynomial in the size of $\mathcal{O}$, and the lower bound follows again from the reduction in [13] and ExPTime-hardness of consistency in classical $\mathcal{A L C}$ [29].

To the best of our knowledge, it is still open whether consistency in $\mathcal{S R O I}$ and $\mathcal{S R O Q}$ is 2-ExpTIME-hard, even in the classical case [17,28]; the best known lower bound is the ExPTIME-hardness of $\mathcal{A L C}$ [29]. We also leave open the complexity of $\mathrm{G}-\mathcal{S H O Q}$, which is ExpTIME-complete in the classical case [17,29].

\section{Conclusions}

Using a combination of techniques developed for infinitely valued Gödel extensions of $\mathcal{A L C}[12]$ and for finitely valued Gödel extensions of $\mathcal{S R O I Q}[6,7,14,15]$, we derived several tight complexity bounds for consistency in sublogics of G$\mathcal{S R O I Q}$. Our reduction circumvents the best-case exponential behavior of the automata-based approach in [12] and avoids the exponential blowup in the nesting depth of concepts of the reductions in [6,7]. However, it introduces an exponential blowup in the size of the binary encoding of numbers in number restrictions and the number of individual names occurring in the ontology. Beyond the complexity results, an important benefit of our approach is that it does not need 
the development of a specialized fuzzy DL reasoner, but can use any state-ofthe-art reasoner for classical $\mathcal{A} \mathcal{L C O Q}$. For that reason, this new reduction aids in closing the gap between efficient classical and fuzzy DL reasoners.

A promising direction for future research is to integrate our reduction directly into a classical tableaux procedure. Observe that the axioms in $\operatorname{red}(C)$ are already closely related to the rules employed in (classical and fuzzy) tableaux algorithms (see, e.g. [3,8,23]). For example, the concept $\forall r . C$ in a node leads to the creation of an $r$-successor node that witnesses the value of $\forall r . C$, i.e., that satisfies the inequations in $\operatorname{red}(\forall r . C)$. Such a tableaux procedure would need to deal with total preorders in each node, possibly using an external solver.

On the theoretical side, we want to prove 2-NEXPTIME-completeness of reasoning in G-SROIQ . As a prerequisite, we would have to eliminate the dependency on the forest-shaped structure of interpretations. It may be possible to adapt the tableaux rules from [22] for this purpose. It also remains open whether consistency in G-SHOQ is ExPTIME-complete, as for its classical counterpart.

As done in [7], we can also combine our reduction with the one for infinitely valued Zadeh semantics. While not based on a t-norm, it is one of the most widely used semantics for fuzzy applications. It also shares many properties of the classical semantics, and hence is a natural choice for simple applications.

\section{References}

1. Baader, F., Calvanese, D., McGuinness, D.L., Nardi, D., Patel-Schneider, P.F. (eds.): The Description Logic Handbook: Theory, Implementation, and Applications. Cambridge University Press, 2nd edn. (2007)

2. Baader, F., Peñaloza, R.: On the undecidability of fuzzy description logics with GCIs and product t-norm. In: Proc. FroCoS'11, LNCS, vol. 6989, pp. 55-70. Springer (2011)

3. Baader, F., Sattler, U.: An overview of tableau algorithms for description logics. Studia Logica 69(1), 5-40 (2001)

4. Bobillo, F., Delgado, M., Gómez-Romero, J.: A crisp representation for fuzzy $\mathcal{S H O I N}$ with fuzzy nominals and general concept inclusions. In: Uncertainty Reasoning for the Semantic Web I. LNAI, vol. 5327, pp. 174-188. Springer (2008)

5. Bobillo, F., Delgado, M., Gómez-Romero, J.: Optimizing the crisp representation of the fuzzy description logic $\mathcal{S R O} \mathcal{I} \mathcal{Q}$. In: Uncertainty Reasoning for the Semantic Web I. LNAI, vol. 5327, pp. 189-206. Springer (2008)

6. Bobillo, F., Delgado, M., Gómez-Romero, J., Straccia, U.: Fuzzy description logics under Gödel semantics. Int. J. Approx. Reason. 50(3), 494-514 (2009)

7. Bobillo, F., Delgado, M., Gómez-Romero, J., Straccia, U.: Joining Gödel and Zadeh fuzzy logics in fuzzy description logics. Int. J. Uncertain. Fuzz. 20(4), 475-508 (2012)

8. Bobillo, F., Straccia, U.: Fuzzy description logics with general t-norms and datatypes. Fuzzy Set. Syst. 160(23), 3382-3402 (2009)

9. Bobillo, F., Straccia, U.: Reasoning with the finitely many-valued Łukasiewicz fuzzy

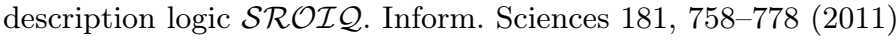

10. Bobillo, F., Straccia, U.: Finite fuzzy description logics and crisp representations. In: Uncertainty Reasoning for the Semantic Web II, LNCS, vol. 7123, pp. 102-121. Springer (2013) 
11. Borgwardt, S.: Fuzzy DLs over finite lattices with nominals. In: Proc. DL'14. CEUR-WS, vol. 1193, pp. 58-70 (2014)

12. Borgwardt, S., Distel, F., Peñaloza, R.: Decidable Gödel description logics without the finitely-valued model property. In: Proc. KR'14. pp. 228-237. AAAI Press (2014)

13. Borgwardt, S., Distel, F., Peñaloza, R.: The limits of decidability in fuzzy description logics with general concept inclusions. Artif. Intell. 218, 23-55 (2015)

14. Borgwardt, S., Peñaloza, R.: The complexity of lattice-based fuzzy description logics. J. Data Semant. 2(1), 1-19 (2013)

15. Borgwardt, S., Peñaloza, R.: Finite lattices do not make reasoning in $\mathcal{A L C O I}$ harder. In: Uncertainty Reasoning for the Semantic Web III, LNAI, vol. 8816, pp. 122-141. Springer (2014)

16. Borgwardt, S., Peñaloza, R.: Infinitely valued Gödel semantics for expressive description logics. LTCS-Report 15-11, Chair for Automata Theory, TU Dresden, Germany (2015), see http://lat.inf.tu-dresden.de/research/reports.html.

17. Calvanese, D., Eiter, T., Ortiz, M.: Regular path queries in expressive description logics with nominals. In: Proc. IJCAI'09. pp. 714-720. AAAI Press (2009)

18. Cerami, M., Straccia, U.: On the (un)decidability of fuzzy description logics under Łukasiewicz t-norm. Inform. Sciences 227, 1-21 (2013)

19. Droste, M., Kuich, W., Vogler, H.: Handbook of Weighted Automata. Springer, 1st edn. (2009)

20. Hájek, P.: Metamathematics of Fuzzy Logic (Trends in Logic). Springer (2001)

21. Hájek, P.: Making fuzzy description logic more general. Fuzzy Set. Syst. 154(1), $1-15(2005)$

22. Horrocks, I., Kutz, O., Sattler, U.: The even more irresistible $\mathcal{S} \mathcal{R O} \mathcal{I} \mathcal{Q}$. In: Proc. KR'06. pp. 57-67. AAAI Press (2006)

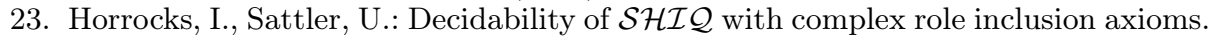
Artif. Intell. 160(1-2), 79-104 (2004)

24. Horrocks, I., Sattler, U., Tobies, S.: Practical reasoning for very expressive description logics. L. J. IGPL 8(3), 239-263 (2000)

25. Kazakov, Y.: A polynomial translation from the two-variable guarded fragment with number restrictions to the guarded fragment. In: Proc. JELIA'04. LNCS, vol. 3229, pp. 372-384. Springer (2004)

26. Kazakov, Y.: $\mathcal{R} \mathcal{I} \mathcal{Q}$ and $\mathcal{S} \mathcal{R O} \mathcal{I} \mathcal{Q}$ are harder than $\mathcal{S H O} \mathcal{I}$. In: Proc. KR'08. pp. 274-284. AAAI Press (2008)

27. Klement, E.P., Mesiar, R., Pap, E.: Triangular Norms. Trends in Logic, Studia Logica Library, Springer (2000)

28. Ortiz, M., Šimkus, M.: Reasoning and query answering in description logics. In: Reasoning Web. 8th Int. Summer School, LNCS, vol. 7487, chap. 1, pp. 1-53. Springer (2012)

29. Schild, K.: A correspondence theory for terminological logics: Preliminary report. In: Proc. IJCAI'91. pp. 466-471. Morgan Kaufmann (1991)

30. Schmidt-Schauß, M., Smolka, G.: Attributive concept descriptions with complements. Artif. Intell. 48(1), 1-26 (1991)

31. Straccia, U.: Transforming fuzzy description logics into classical description logics. In: Proc. JELIA'04. LNCS, vol. 3229, pp. 385-399. Springer (2004)

32. Tobies, S.: The complexity of reasoning with cardinality restrictions and nominals in expressive description logics. J. Artif. Intell. Res. 12, 199-217 (2000)

33. Tobies, S.: Complexity Results and Practical Algorithms for Logics in Knowledge Representation. Ph.D. thesis, RWTH Aachen, Germany (2001) 